REVIEW

Published July 21, 2018

\title{
Abstracts from the May 2018 Cleveland Immunopathogenesis Consortium Meeting
}

Corresponding Author

Michael M. Lederman

Case Western Reserve University, Cleveland, Ohio

MXL6@case.edu
DOI

10.20411/pai.v3i1.254

\section{SUGGESTED CITATION}

Lederman MM. Abstracts from the May 2018 Cleveland Immunopathogenesis Consortium Meeting. Pathogens and Immunity. 2018;3(1):114-25. doi: 10.20411/pai.v3i1.254

Below are selected abstracts from presentations at the annual Cleveland Immunopathogenesis Consortium meeting that was held on the campus of Case Western Reserve University in May 2018. The presentations covered a range of topics from HIV persistence/eradication to coinfection, inflammation and its determinants, and immunologic failure. These abstracts are provided by Pathogens and Immunity to inform interested readers about these findings. You are encouraged to contact the lead authors of these works to learn more. Their email addresses are at the end of each abstract.

\section{RHEUMATOID FACTOR LEVELS REMAIN PERSISTENTLY ELEVATED 24 WEEKS (AND BEYOND) AFTER IFN-FREE HCV THERAPY IN THE MAJORITY OF RF+ HCV INFECTED PERSONS}

\section{Authors}

Corinne Kowal, Carey Shive, Elizabeth Zebrowski, Lenche Kostadinova, Brianna Fuller, Elane Reyes, Kelsey Rife, Amy Hirsch, Anita Compan, Yngve Falck-Ytter, Daniel L. Popkin, Leonard H. Calabrese, Maya Mattar, Donald D. Anthony 


\section{Lead Author}

Donald D. Anthony

Lead Author's Institution

Case Western Reserve University, Cleveland Clinic, and VA Medical Center, Cleveland, Ohio

\section{Summary}

HCV Cryoglobulinemic vasculitis has been observed in some cases to linger long after Interferon free direct acting antiviral (DAA) HCV therapy induced sustained virologic response (SVR). Cryoglobulins are one pathogenic factor involved in this vasculitis. They are composed in part of Rheumatoid Factor (RF). Here we observed that RF remains positive (elevated) in the majority of RF+ HCV infected patients over 24 weeks after initiation of successful IFN free therapy. This variability in resolution of this immune derangement after successful treatment of HCV has implications for time to clinical improvement in HCV associated Cryoglobulinemic vasculitis, and provides support for a model where factors other than HCV itself participate in determining RF level during chronic active HCV infection, and during IFN free DAA therapy.

\section{Lead Author's Contact Information}

dda3@case.edu

\section{MAINTENANCE OF THE GUT INTEGRITY RATHER THAN THE CONTROL OF T CELL ACTIVATION DRIVES CONTROL OF DISEASE PROGRESSION IN AFRICAN GREEN MONKEYS}

\section{Authors}

Cristian Apetrei, Kevin D. Raehtz, Kathryn J. Martin, Noah Krampe, Tianyu He, Egidio Brocca-Cofano, Ruy M. Ribeiro, Ivona Pandrea

\section{Lead Author}

Cristian Apetrei

\section{Lead Author's Institution}

Center for Vaccine Research, University of Pittsburgh, Pittsburgh, Pennsylvania

\section{Summary}

We report here for the first time that natural hosts of SIVs (i.e., the African green monkeys) have the ability to maintain the integrity of the mucosal barrier throughout SIV infection. Furthermore, by dissociating $\mathrm{T}$ cell activation from mucosal and systemic inflammation, through regulatory $\mathrm{T}$ cell depletion, we showed that maintenance of mucosal integrity rather than the resolution of $\mathrm{T}$ cell activation at the transition from acute-to-chronic infection is the main factor behind the ability of natural hosts to avoid disease progression.

\section{Lead Author's Contact Information}

apetreic@pitt.edu 


\section{HIV-ASSOCIATED CARDIOVASCULAR DISEASE IN THE GLOBAL CONTEXT: MOVING FROM CORONARY ARTERY TO THE MYOCARDIUM}

\section{Authors}

Jason V. Baker, Lye-Yeng Wong, Tess Peterson, Justin Lumbamba, Ntobeko Ntusi, Mpiko Ntsekhe

\section{Lead Author}

Jason V. Baker

\section{Lead Author's Institution}

Hennepin Healthcare Research Institute, Minneapolis, Minnesota

\section{Summary}

HIV associated cardiovascular disease (HIV-CVD) has been characterized by accentuated atherogenesis mediated, in part, by activation of innate immunity involving monocyte-macrophage pathways. Data describing this pathogenesis has been primarily ascertained in high-income countries, but with expanding antiretroviral coverage the burden of disease from HIV-CVD is also growing in low-to-middle-income countries. The phenotype of HIV-CVD is very likely to manifest differently in low-to-middle-income countries, due to differences in risk factor profiles, but may still involve activation of monocyte-macrophage pathways contributing to myocardial tissue inflammation, fibrosis, and ultimately dysfunction over time.

\section{Lead Author's Contact Information}

baker@umn.edu

\section{Authors}

\section{ALTERED ENTERIC VIROME IN UNTREATED HIV INFECTION}

Celia Boukadida, Sandra Pinto-Cardoso, Ana Paola Carranco-Arenas, Monserrat Chávez-Torres, Daniela Garrido-Rodríguez, Amy Peralta-Prado, Gustavo Reyes-Terán

\section{Lead Author}

Celia Boukadida

\section{Lead Author's Institution}

Departamento de Investigación en Enfermedades Infecciosas, Instituto Nacional de Enfermedades Respiratorias, Mexico City, Mexico

\section{Summary}

Advanced HIV infection was associated with a decrease in the abundance of plant viruses in stool samples and with an increase in the prevalence, abundance and diversity of human viruses. In particular, low peripheral CD4 T cell counts were associated with a striking expansion of viral sequences from the Anelloviridae family, commensal viruses recently suggested to reflect the overall state of immunosuppression. The expansion of human viruses might contribute to GI tract damage and persistent inflammation in immunocompromised HIV-infected individuals.

\section{Lead Author's Contact Information \\ celia.boukadida@cieni.org.mx}




\section{TRANSCRIPTOMIC APPROACHES TO IDENTIFYING POTENTIAL MECHANISMS OF ELITE CONTROL IN HIV INFECTION}

\section{Authors}

Cheryl Cameron, Brian Richardson, Jackelyn Golden, Aarthi Talla, Remi Fromentin, Mary Ann Checkley, Ben Luttge, Krupa Subramanian, Peter Stock, Jean-Pierre Routy, Cecile Tremblay, Julie McElrath, Tim Henrich, Deanna Kulpa, Nicolas Chomont, Jonathan Karn, Steven Deeks, Mark Cameron, Rafick Sekaly

\section{Lead Author}

Cheryl Cameron

\section{Lead Author's Institution}

Case Western Reserve University, Cleveland, Ohio

\section{Summary}

We reported results from studies designed to identify potential therapeutic targets that could lead to or support a functional cure of HIV. We applied a comparative transcriptomic analysis of CD8 and NK cell subsets from elite controllers and CART suppressed subjects to identify potential mechanisms that allow elite controllers to maintain aviremic status in the absence of antiretroviral therapy. Newly identified pathways associated with innate aviremic status were validated and further dissected by immunological assays.

\section{Lead Author's Contact Information}

cheryl.cameron@case.edu

\section{POTENT AND SELECTIVE KILLING OF LATENT HIV-INFECTED MEMORY T CELLS BY NK CELL EXPANDED EX VIVO}

\section{Authors}

Mary Ann Checkley Luttge, Benjamin Luttge, Curtis Dobrowolski, Jonathan Karn

\section{Lead Author}

Mary Ann Checkley Luttge

\section{Lead Author's Institution}

Case Western Reserve University, Cleveland, Ohio

\section{Summary}

We are pursuing a novel "kick and kill" HIV eradication strategy whereby treatment of patients with latency reversing agents would be followed by the adoptive transfer of autologous eNK cells, which are expanded and activated ex vivo prior to reinfusion. We find that eNK cells from HIV+ donors have a highly cytotoxic phenotype that is equal to eNK cells from HIV-negative donors. Using a primary HIV latent T cell model, we demonstrated that eNK cells displayed selective 
killing of latently HIV-infected memory T cells after proviral reactivation. Finally, eNK cells from HIV+ participants efficiently kill autologous HIV+ Tm cells after proviral reactivation with vorinostat and IL-15 as detected by a progressive loss of inducible cell-associated HIV mRNA for up to at least seven days.

\section{Lead Author's Contact Information}

mxc567@case.edu

\section{COMBINING NON-INVASIVE IMAGING APPROACH AND TISSUE ANALYSES TO ASSESS LN DRAINING FUNCTION}

\section{Authors}

Claire Deleage, Baris Turkbey, Catherine Brands, David Morcock, Marcelino L. Bernardo, Hyun Jung, Yanling Liu, Gregory Q. Del Prete, Brandon F. Keele, Jeffrey D. Lifson, Jacob D. Estes

\section{Lead Author}

Claire Deleage

\section{Lead Author's Institution}

AIDS and Cancer Virus program - Frederick National Laboratory for Cancer Research, Frederick, Maryland

\section{Summary}

We used longitudinal magnetic resonance imaging (MRI) to study uptake of a simulated particulate antigen by draining lymph nodes in SIV infected rhesus macaques and correlated these findings with histopathological analysis. We found that SIV infection is associated with a profound impairment of LN draining function as soon as 2 weeks post infection. We think that the relocation and persistence of DC and CD169+ cells within the subcapsular region, capturing the antigens, may affect the ability to mount effective immune responses.

\section{Lead Author's Contact Information}

claire.deleage@nih.gov

\section{MACROPHAGE ACTIVATION: A POTENTIAL CONTRIBUTOR TO CVD RISK IN HIV INFECTION?}

\section{Authors}

Emily Bowman, Brian Richardson, Sue Koletar, Mark Cameron, Nicholas T. Funderburg

\section{Lead Author}

Nicholas T. Funderburg

\section{Lead Author's Institution}

Ohio State University, School of Health and Rehabilitation Sciences, Columbus, Ohio

\section{Summary}

Monocytes can migrate from the bloodstream and into the blood vessel wall where they differentiate into macrophages and produce several inflammatory mediators. We have demonstrated previously that monocytes from people living with HIV (PLWH) are activated and express in- 
creased vascular homing receptors compared to cells from HIV- individuals. We now report that monocyte derived macrophages from PLWH have altered gene expression profiles and produce increased levels of inflammatory cytokines, reactive oxygen species, and matrix metalloproteases compared to findings in cells from HIV- individuals.

\section{Lead Author's Contact Information}

Nicholas.Funderburg@osumc.edu

\section{THE LAST GIFT: \\ PERFORMING HIV CURE RESEARCH AT THE END OF LIFE}

\section{Authors}

Sara Gianella, Davey Smith

\section{Lead Author}

Sara Gianella

\section{Lead Author's Institution}

University of California, San Diego, San Diego, California

\section{Summary}

To cure HIV, we need a better understanding of the distribution of the HIV reservoirs throughout the body and how these reservoirs contribute to viral rebound after discontinuation of antiretroviral therapy (ART). Extensive investigations in humans have been unable to fully characterize the large and complex HIV reservoirs that must be eradicated to achieve a cure, because biological sampling is necessarily limited in life. To take the next steps in understanding and eradicating HIV reservoirs in tissues, we developed a "peri-mortem translational research model" (http://lastgift.ucsd.edu/), similar to existing models in cancer research. In this model, altruistic individuals living with HIV, with advanced non-AIDS related diseases and with six months or less to live, are participating in HIV cure research. These altruistic individuals will provide: (i) detailed clinical, risk and socio-demographic information before their death; (ii) weekly blood collections while they are alive and, (iii) their entire bodies after they die for a rapid autopsy ( $<6 \mathrm{~h}$ from the time of death). In these volunteers, we will characterize cellular and tissue populations of HIV in blood (ante-mortem) and compare these populations in blood and anatomic compartments (at autopsy) while on and off ART to better understand dynamics of both viral rebound and populations in the blood and throughout the body. These altruistic individuals will provide a wealth of clinical information and biological specimens that can be used to answer important unanswered questions in the quest to cure HIV.

\section{Lead Author's Contact Information}

gianella@ucsd.edu

\section{PATHOLOGICAL ROLE OF ANTI-CD4 ANTIBODIES IN HIV-INFECTED IMMUNOLOGIC NONRESPONDERS RECEIVING VIRUS-SUPPRESSIVE ANTIRETROVIRAL THERAPY}

\section{Authors}

Zhenwu Luo, Zhen Li, Lisa Martin, Zhuang Wan, Eric G. Meissner, Enrique Espinosa, Hao Wu, 
Xiaocong Yu, Pingfu Fu, Maria Anna Julia Westerink, J. Michael Kilby, Jennifer Wu, Lei Huang, Sonya L. Heath, Zihai Li, Wei Jiang

\section{Lead Author}

Wei Jiang

\section{Lead Author's Institution}

Medical University of South Carolina, Charleston, South Carolina

\section{Summary}

Increased mortality and morbidity occur among human immunodeficiency virus (HIV)-infected patients in whom CD4+ T-cell counts do not increase despite viral suppression with antiretroviral therapy (ART). Here we identified an underlying mechanism. Significantly elevated plasma levels of anti-CD4 immunoglobulin G (IgG) were found in HIV-positive immunologic nonresponders (HIV-positive individuals with CD4+ T-cell counts of $\leq 350$ cells/ $\mu \mathrm{L}$ ), compared with levels in HIV-positive immunologic responders (HIV-positive individuals with CD4+ T-cell counts of $\geq 500$ cells $/ \mu \mathrm{L}$ ) and healthy controls. Higher plasma level of anti-CD4 IgG correlated with blunted CD4+ T-cell recovery. Furthermore, purified anti-CD4 IgG from HIV-positive immunologic nonresponders induced natural killer (NK) cell-dependent CD4+ T-cell cytolysis and apoptosis through antibody-dependent cell-mediated cytotoxicity (ADCC) in vitro. These data indicate that autoreactive anti-CD4 IgG may play an important role in blunted CD4+ T-cell reconstitution despite effective ART.

\section{Lead Author's Contact Information}

jianw@musc.edu

\section{Authors}

\section{RESERVOIR DOGGEREL}

Jonathan Karn, Curtis Dobrowolski, Ben Luttge, Tim Shacker, Miguel Quinones

\section{Lead Author}

Jon Karn

\section{Lead Author's Institution}

Case Western Reserve University, Cleveland, Ohio

\section{Summary}

New assays to more accurately measure HIV reservoirs were developed using next generation sequencing technologies and digital droplet PCR. Intact proviral genomes were estimated using a two color ddPCR assay with primers targeting the gag and env regions of the genome. The EDITS assay measured inducible env RNA, a late gene product whose splicing pattern samples a large region of the genome. EDITS can detect single cells expressing HIV RNA and has an accuracy of plus minus $10 \%$. The assay has been used to monitor induction of HIV following treatment of patients with ALT803 (an IL-15 derivative). It has also been adapted to measure replication competent virus at very early time points following proviral induction ex vivo. 


\section{Lead Author's Contact Information}

jxk153@case.edu

\section{HOW OFTEN CAN YOU GO TO THE WELL: HIV COMPARTMENTALIZATION DURING OPPORTUNISTIC INFECTIONS: PRE-ART CRYPTOCOCCAL MENIGNITIS}

\section{Authors}

Maura Manion, Camille Lange, Kathy Huppler Hullsiek, Brandon Keele, Helene Highbarger, David Meya, David Boulware, Frank Maldarelli, Irini Sereti

\section{Lead Author}

Maura Manion

\section{Lead Author's Institution}

National Institute of Allergy and Infectious Diseases, Bethesda, Maryland

\section{Summary}

Characterization of HIV populations in the CSF and blood compartments in HIV patients with cryptococcal meningitis pre-ART.

\section{Lead Author's Contact Information}

maura.manion@nih.gov

\section{Authors}

Wendy Fitzgerald, Michael L. Freeman, Michael M. Lederman, Elena Vasilieva, Roberto Romero, Leonid Margolis

\section{Lead Author}

Leonid Margolis

\section{Lead Author's Institution}

Section of Intercellular Interactions, Eunice Kennedy-Shriver National Institute of Child Health and Human, Development, National Institutes of Health, Bethesda, Maryland

\section{Summary}

Cytokines that are considered to be soluble mediators of cell-cell interactions, have been found in various in vitro, ex vivo and in vivo systems to be associated with extracellular vesicles, both being presented on the vesicles' surface and encapsulated. Association with extracellular vesicles is a function of a biological system and is changed upon systems' activation.

\section{Lead Author's Contact Information}

margolis@helix.nih.gov 


\section{IL-15 DRIVES THE GENERATION AND SURVIVAL OF SENESCENT CD8 T CELLS IN HIV/CMV CO-INFECTION}

\section{Authors}

Stephen R. Morris, Bonnie Chen, Soumya Panigrahi, Scott F. Sieg, Nicholas T. Funderburg, Souheil-Antoine Younes, Sara Gianella, Michael M. Lederman, Michael L. Freeman

\section{Lead Author}

Stephen R. Morris

\section{Lead Author's Institution}

Case Western Reserve University, Cleveland, Ohio*

\section{Summary}

We show that HIV/CMV co- infection and related inflammation can drive maturation of memory CD8 T cells toward an "inflammescent" phenotype that can localize to atherosclerotic plaques and has surface markers of senescence. Our in vitro data suggest that these ostensibly senescent CD57+ CX3CR1+ CD8 T cells can survive and proliferate in an Ag-independent manner, via IL-15 stimulation, which supported mitochondrial activity, as well as survival and proliferation signals.

\section{Lead Author's Contact Information \\ srmorris77@hotmail.com \\ * Current institution: University of Miami, Miami, Florida}

\section{"BREAKING BAD"}

\section{Authors}

Joseph Mudd, Cheryl Cameron, Mark Cameron, Jason Brenchley

\section{Lead Author}

Joseph Mudd

\section{Lead Author's Institution}

National Institute of Allergy and Infectious Diseases, National Institutes of Health, Bethesda, Maryland

\section{Summary}

The focus of this work was aimed at understanding the mechanisms of CD4 downregulation in African green monkeys, who are natural hosts of SIV infection. In genome-wide screening of AGM CD4 T cells induced to downregulate CD4, we found that the DNA methylation machinery is differentially regulated in these cells, and we can block CD4 downregulation with inhibitors of DNA methylating enzymes.

\section{Lead Author's Contact Information}

joseph.mudd@nih.gov 


\section{IL-10 LEVELS ARE INDUCED AFTER SIV INFECTION, NOT NORMALIZED BY ART, AND REGULATE VIRAL PERSISTENCE IN SIV-INFECTED NONHUMAN PRIMATES}

Authors

Justin Harper, Susan Ribeiro, Rafick Sekaly, Jacob Estes, Mirko Paiardini

Lead Author

Mirko Paiardini

Lead Author's Institution

Emory University, Emory University School of Medicine, Yerkes National Primate Research Center, Atlanta, Georgia

\section{Summary}

The mechanisms regulating the establishment and maintenance of the HIV reservoir are unknown, thus limiting the design of therapeutic strategies to limit it. We found that plasma interleukin-10 becomes elevated upon SIV-infection and correlates with measures of disease progression in chronic infection, such as CD4 counts and immune activation levels. Furthermore, plasma levels of IL-10 fails to normalize under suppressive ART and correlate with the frequency of CD4+ T follicular helper cells and SIV-DNA content in blood and lymph node during ART. Thus, IL-10 contributes to viral persistence in ART-treated, SIV-infected nonhuman primates.

Lead Author's Contact Information

mirko.paiardini@emory.edu

\section{Authors}

\section{IMMUNE RECONSTITUTION AFTER INITIATION OF ANTIRETROVIRAL THERAPY}

Sandra Pinto-Cardoso, Monserrat Chávez-Torres, Amy Peralta-Prado, Gustavo Reyes-Terán

\section{Lead Author}

Sandra Pinto-Cardoso

\section{Lead Author's Institution}

Departamento de Investigación en Enfermedades Infecciosas, Instituto Nacional de Enfermedades Respiratorias, Ciudad de México

\section{Summary}

The longitudinal analysis of the gut microbiome of HIV-infected individuals starting their first antiretroviral treatment showed that certain gut bacteria become activated with significant increased fold-changes in the genus Fusobacterium, a known oral commensal/pathobiont and the genus Succinivibrio belonging to the Proteobacteria phylum. Notably, we found a decrease in Enterobacteriaceae family. No significant fold-change in Firmicutes was observed (both Prevotella and Bacteroides genera remain fairly constant). Bacterial diversity was also very dynamic within the first year of ART (gain and loss) but overall did not increase significantly after 1 year on ART.

\section{Lead Author's Contact Information}

sandra.pinto@cieni.org.mx 


\section{HIV, AGING, AND IMMUNE FAILURE}

\section{Authors}

Carey Shive, Corinne Kowal, Dave Canaday, Michael M. Lederman, Benigno Rodriguez, Donald Anthony

\section{Lead Author}

Carey Shive

\section{Lead Author's Institution}

Cleveland VA Medical Center and Department of Pathology, Case Western Reserve University, Cleveland, Ohio

\section{Summary}

Plasma levels of IL-6 are strongly associated with age in healthy and treated, HIV-infected participants. Phenotypically naïve T cells (CD27+ CD45RA+) expressing exhaustion and senescent markers may be memory $\mathrm{T}$ cells with a naïve phenotype $\left(\mathrm{T}_{\mathrm{MNP}}\right)$.

\section{Lead Author's Contact Information}

carey.shive@case.edu

\section{Authors}

\section{UPDATES IN ICL PATHOGENESIS}

Ainhoa Perez-Diez, Chun-Shu Wong, Xiandong Liu, Harry Mystakelis, Jeffrey Bourgeois, Virginia Sheikh, Andrea Lisco, Cornelia Cudricci, Afroditi Boulougoura, Alexandra Freeman, Megan Anderson, Gregg Robby, Peter Burbelo, Peter Williamson, John Tsang, Richard Siegel, Irini Sereti

\section{Lead Author}

Irini Sereti

\section{Lead Author's Institution}

National Institute of Allergy and Infectious Diseases, National Institutes of Health, Bethesda, Maryland

\section{Summary}

An update on pathogenesis of ICL CD4 lymphopenia was presented with a focus on patients presenting with auto-antibodies and autoimmunity.

\section{Lead Author's Contact Information}

ISereti@niaid.nih.gov

\section{IL-15 INDUCES MITOCHONDRIAL BIOGENESIS AND RESCUES IMMUNE EXHAUSTION OF CD4 T CELLS IN HIV-1 INFECTED IMMUNE FAILURE SUBJECTS}

\section{Authors}

Souheil-Antoine Younes, Aarthi Talla, Susan Pereira Ribeiro, Evgeniya V. Saidakova, Larisa B. Korolevskaya, Konstantin V. Shmagel, Carey L. Shive, Michael L. Freeman, Soumya Panigrahi,Sophia Zweig, Robert Balderas, Leonid Margolis, Daniel C. Douek, Donald. D. Anthony, Pushpa 
Pandiyan, Mark Cameron, Scott F. Sieg, Leonard Calabrese, Benigno Rodriguez, Michael M. Lederman

Lead Author

Souheil Younes

Lead Author's Institution

Case Western Reserve University, Cleveland, Ohio

\section{Summary}

In this work we provide evidence that CD4 T cells of immune failure HIV-1 infected persons on ART have a mitochondrial defect linked to low CD4 T cell numbers. This mitochondrial dysfunction is correctible by exposure to IL-15 in vitro.

Lead Author's Contact Information

sxy292@case.edu

FOOTNOTES

Submitted July 20, 2018 | Accepted July 20, 2018 | Published July 21, 2018

\section{COPYRIGHT}

Copyright (C) 2018

This is an open-access article distributed under the terms of the Creative Commons Attribution 4.0 International License. 